\title{
Potassium Hyaluronate
}

National Cancer Institute

\section{Source}

National Cancer Institute. Potassium Hyaluronate. NCI Thesaurus. Code C1445.

The potassium salt form of hyaluronic acid, a glucosaminoglycan, a component of connective tissue, skin, vitreous humour, umbilical cord, synovial fluid and the capsule of certain microorganisms contributing to adhesion, elasticity, and viscosity of extracellular substances. 